This item was submitted to Loughborough's Research Repository by the author.

Items in Figshare are protected by copyright, with all rights reserved, unless otherwise indicated.

\title{
Periodic continued fractions and hyperelliptic curves
}

PLEASE CITE THE PUBLISHED VERSION

LICENCE

CC BY-NC-ND 4.0

\section{REPOSITORY RECORD}

Grosset, Marie-Pierre J.E., and A.P. Veselov. 2019. "Periodic Continued Fractions and Hyperelliptic Curves". figshare. https://hdl.handle.net/2134/2714. 


\title{
PERIODIC CONTINUED FRACTIONS AND HYPERELLIPTIC CURVES
}

\author{
M-P. GROSSET AND A.P. VESELOV
}

Abstract. We investigate when an algebraic function of the form $\phi(\lambda)=$ $\frac{-B(\lambda)+\sqrt{R(\lambda)}}{A(\lambda)}$, where $R(\lambda)$ is a polynomial of odd degree $N=2 g+1$ with coefficients in $\mathbb{C}$, can be written as a periodic $\alpha$-fraction of the form

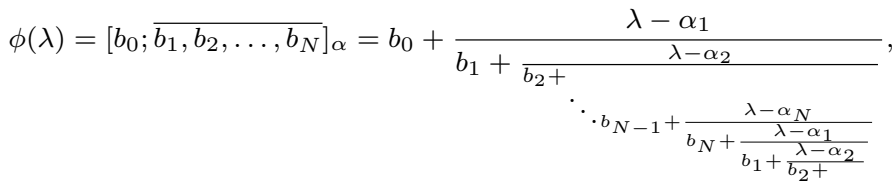

$$
\begin{aligned}
& \ddots
\end{aligned}
$$

for some fixed sequence $\alpha_{i}$. We show that this problem has a natural answer given by the classical theory of hyperelliptic curves and their Jacobi varieties. We also consider pure periodic $\alpha$-fraction expansions corresponding to the special case when $b_{N}=b_{0}$.

\section{INTRODUCTION}

Consider the following continued fraction, which we will call $\alpha$-fractions:

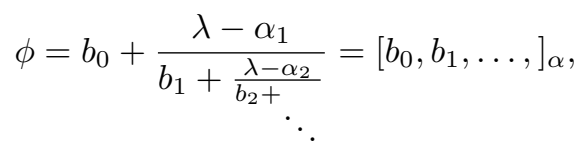

where $\alpha=\left(\alpha_{i}\right), \alpha_{i} \in \mathbb{C}$ is a given sequence, $b_{i}$ are arbitrary complex numbers, $\lambda$ is a formal parameter. In this paper we will consider a special case of $N$-periodic $\alpha$-fractions, when the sequences $\alpha_{i}$ and $b_{i}$ are periodic with period $N$ :

$$
\alpha_{i+N}=\alpha_{i}, b_{i+N}=b_{i}
$$

for all $i \geq 1$ :

$$
\phi=\left[b_{0} ; \overline{b_{1}, b_{2}, \ldots, b_{N}}\right]_{\alpha} .
$$

In the particular case when $b_{N}=b_{0}$ we have $\phi=\left[\overline{b_{0}, b_{1}, \ldots, b_{N-1}}\right]_{\alpha}$, which will be called a pure $N$-periodic $\alpha$-fraction.

This kind of fractions naturally appear in the theory of integrable systems, in particular in the theory of periodic dressing chain [1], but to the best of our knowledge has not been studied so far. We were partly inspired by our recent discussions with Vassilis Papageorgiou on the discrete $\mathrm{KdV}$ equation where such continued fractions appear as well [2]. 
Because of periodicity we can write formally (2) as

$$
\phi=b_{0}+\frac{\lambda-\alpha_{1}}{b_{1}+\frac{\lambda-\alpha_{2}}{b_{2}+\ddots_{b_{N-1}+\frac{\lambda-\alpha_{N}}{b_{N}-b_{0}+\phi}}}},
$$

which implies a quadratic relation

$$
A(\lambda) \phi^{2}+2 B(\lambda) \phi+C(\lambda)=0
$$

where $A, B, C$ are certain polynomials in $\lambda$ with coefficients polynomially depending on $b_{i}$. Thus to any periodic $\alpha$-fraction (2) corresponds an algebraic function

$$
\phi(\lambda)=\frac{-B(\lambda)+\sqrt{R(\lambda)}}{A(\lambda)},
$$

where

$$
R(\lambda)=B(\lambda)^{2}-A(\lambda) C(\lambda)
$$

is the discriminant of (3). In that case we will say that (2) is a periodic $\alpha$-fraction expansion of the algebraic function (4) from the hyperelliptic extension $\mathbb{C}(\lambda, \sqrt{R(\lambda)})$ of the field of rational functions $\mathbb{C}(\lambda)$. We leave the question of convergence aside concentrating on algebraic and geometric aspects of the problem.

We will discuss the following three main questions.

Question 1. Which algebraic functions (4) admit $N$-periodic $\alpha$-fraction expansions?

Question 2. How many such expansions may exist for a given algebraic function (4) and how to find them ?

Question 3. What is the geometry of the set of functions (4) from given hyperelliptic extension (i.e. with fixed $R$ ), which admit periodic $\alpha$-fraction expansions?

The answers depend on the parity of $N$. In this paper we will restrict our study to the case of odd period $N=2 g+1$, which is the most interesting one (cf. [1]). We will also assume that all the parameters $\alpha_{i}$ are distinct.

Note that when $N$ (which is also the degree of $R(\lambda)$ ) is even, one can consider the usual continued fraction expansions going back to Abel and Chebyshev who discovered their relation with the classical problem of integration in elementary functions (see a nicely written paper by van der Poorten and Tran [3] for details). These expansions are more natural, but can not be used in the odd degree case.

We would like to mention also that in the classical number-theoretic version the answer to Question 1 is due to Galois, who proved that a quadratic irrationality $\xi=p+q \sqrt{d}, p$ and $q$ rational numbers, $d$ integer, has a pure periodic continued fraction expansion $\xi=\left[\overline{a_{0}, \ldots, a_{k}}\right]$ if and only if $\xi$ is larger than 1 and its conjugate $\bar{\xi}=p-q \sqrt{d}$ lies between -1 and 0 (see e.g. [4]). Note also that the periodic continued fractions of the form $\left[a_{0} ; \overline{a_{1}, \ldots, a_{k}}\right]$ also appear naturally in number theory as expansions of $\sqrt{d}$ (see [4]).

To explain our main results let us introduce the polynomial

$$
\mathfrak{A}(\lambda)=\prod_{i=1}^{N}\left(\lambda-\alpha_{i}\right)
$$

and call a polynomial $R$ of degree $N \alpha$-admissible if

$$
R(\lambda)=S^{2}(\lambda)+\mathfrak{A}(\lambda)
$$


for some polynomial $S(\lambda)$ of degree $g$ or less, where as before $N=2 g+1$. We will call a polynomial monic if its highest coefficient is 1 and anti-monic if it is equal to -1 . Note that $\alpha$-admissible polynomials $R$ are automatically monic.

Theorem 1. The algebraic functions $\phi(\lambda)$ admitting an $N$-periodic $\alpha$-fraction expansion have the form $(3,4)$ with the polynomials $A, B, C$ satisfying the following conditions:

(1) $\operatorname{deg} B \leq g, \quad A(\lambda)$ and $C(\lambda)$ are monic and anti-monic polynomials of degree $g$ and $g+1$ respectively

(2) the discriminant $R(\lambda)=B^{2}-A C$ is $\alpha$-admissible.

Conversely, for an open dense subset of such triples $(A, B, C)$ the corresponding function (4) has exactly two $N$-periodic $\alpha$-fraction expansions. The corresponding $b_{i}$ are rational functions of both coefficients of $A, B, C$ and parameters $\alpha_{i}$ and can be found by an effective matrix factorisation procedure.

In the pure periodic case the only additional requirement is

$$
C\left(\alpha_{N}\right)=0,
$$

under which the pure periodic $\alpha$-fraction expansion is generically unique.

We will call $(A, B, C)$ satisfying the conditions (1), (2) of Theorem 1 the $\alpha$-triples. Note that these conditions are invariant under any permutation of the parameters $\alpha_{i}$. In fact there is a natural birational action of the direct product $G=\mathbb{Z}_{2} \times S_{N}$ on the set of periodic continued $\alpha$-fraction expansions, where the generator $\varepsilon$ of $\mathbb{Z}_{2}$ is acting simply by swapping two different $\alpha$-fraction expansions given by Theorem 1. Our next result describes this action explicitly.

Let us introduce the following permutation $\pi \in S_{N}$, which reverses the order $\alpha_{1}, \alpha_{2}, \ldots, \alpha_{N-1}, \alpha_{N}$ to $\alpha_{N}, \alpha_{N-1}, \ldots, \alpha_{2}, \alpha_{1}$ and the involutions $\sigma_{k}$ swapping $\alpha_{k}$ and $\alpha_{k+1}$, where $k=1, \ldots, N-1$.

We will show that $\sigma_{k}$ is acting on $b=\left(b_{i}\right), i=0, \ldots, N$ with $b_{k} \neq 0$ as follows:

$$
\tilde{b}_{k-1}=b_{k-1}+\frac{\alpha_{k+1}-\alpha_{k}}{b_{k}}, \quad \tilde{b}_{k+1}=b_{k+1}-\frac{\alpha_{k+1}-\alpha_{k}}{b_{k}},
$$

the rest of $b_{i}$ remain the same. This determines the action of the symmetric group $S_{N}$ since $\sigma_{k}$ generate it. To describe the action of $\mathbb{Z}_{2}$ it is enough to describe the action of the involution $\varepsilon \pi \in G$, which turns out to be quite simple:

$$
\tilde{b}_{j}=-b_{N-j}, j=1, \ldots, N-1 \quad \tilde{b}_{0}=b_{0}-b_{N}, \quad \tilde{b}_{N}=-b_{N} .
$$

Theorem 2. The formulae (9) and (10) define a birational action of the group $G=$ $\mathbb{Z}_{2} \times S_{N}$ on the set of $N$-periodic $\alpha$-fractions. Its orbits consist of all $2 N$ ! possible periodic $\alpha$-fraction expansions for a given $\alpha$-triple $(A, B, C)$ and any permutation of the parameters $\alpha_{i}$.

In the pure periodic case the symmetry group is broken down to $S_{N-1}$ generated by $\sigma_{k}$ with $k=1, \ldots, N-2$ given by (9).

Let us fix now the $\alpha$-admissible polynomial $R(\lambda)$ with distinct roots. We would like to describe the geometry of the set of elements from the hyperelliptic extension field $\mathbb{C}(\lambda, \sqrt{R(\lambda)})$ which have a pure periodic $\alpha$-fraction expansion. For the standard material from the algebraic geometry of the curves we refer to the classical Griffiths-Harris book [5].

Consider the hyperelliptic curve $\Gamma_{R}$ given by the equation

$$
\mu^{2}=R(\lambda) \text {. }
$$


The curve $\Gamma_{R}$ consists of the affine part $\Gamma_{R}^{a f f}$, corresponding to the "finite" solutions of (11), and the "infinity" point, which we will denote as $P_{\infty}$. Since the roots of $R$ are distinct it is non-singular and has genus $g$. Consider $g$ points $P_{1}, \ldots, P_{g}$ of $\Gamma_{R}^{a f f}$ and call the corresponding divisor $D=P_{1}+\cdots+P_{g}$ non-special if

$$
P_{i} \neq \tau\left(P_{j}\right)
$$

for any $i \neq j$, where $\tau$ is the hyperelliptic involution:

$$
\tau(\mu, \lambda)=(-\mu, \lambda)
$$

Non-special divisors have the property that the linear space $L(D)$ of all meromorphic functions on $\Gamma_{R}$ having the poles at $D$ of order less than or equal to 1 has dimension 1, which means that it consists only of constant functions. The corresponding linear space $L\left(D+P_{\infty}\right)$ has dimension 2 , so there exists a non-constant function $f \in L\left(D+P_{\infty}\right)$ with additional pole at infinity. These functions are in a way "least singular" among the "generic" meromorphic functions on $\Gamma_{R}$ in the sense that any such function can not have less than $g+1$ poles (see [5]).

Now define the affine Jacobi variety $J\left(\Gamma_{R}\right)^{\text {aff }}$ as the set of positive non-special divisors $D=P_{1}+\cdots+P_{g}, P_{1}, \ldots, P_{g} \in \Gamma_{R}^{a f f}$.

Let $M_{R}^{\alpha}$ be an affine variety of $\alpha$-triples of polynomials $(A, B, C)$ with given discriminant $R(\lambda)$, and $P_{R}^{\alpha}$ be its subvariety given by the additional condition (8).

Theorem 3. There exists a bijection between the set $M_{R}^{\alpha}$ and the extended affine Jacobi variety $J\left(\Gamma_{R}\right)^{a f f} \times \mathbb{C}$. The corresponding algebraic functions (4) can be characterised as meromorphic functions $\phi \in L\left(D+P_{\infty}\right)$ on $\Gamma_{R}$ with non-special pole divisor $D+P_{\infty}$ and asymptotic $\phi \sim \sqrt{\lambda}$ at infinity.

In the pure periodic case under the assumption that $R\left(\alpha_{N}\right) \neq 0$ there exists a natural $2: 1$ map from the set $P_{R}^{\alpha}$ to $J\left(\Gamma_{R}\right)^{\text {aff }}$. The corresponding $\phi$ from $L(D+$ $\left.P_{\infty}\right)$ are fixed by the condition that one of two values of $\phi\left(\alpha_{N}\right)$ is zero.

The proof is based on the classical description of the Jacobi variety due to Jacobi himself [6] (see also Mumford [7]).

We see that the affine space $\mathbb{C}^{N}$ of all functions (4) having periodic $\alpha$-fraction expansion is birationally equivalent to the double covering of the bundle of the extended affine Jacobians of $\alpha$-admissible hyperelliptic curves. This is a version of the well-known result by Dubrovin and Novikov [8] who were the first to apply the theory of the $\mathrm{KdV}$ equation to the problems of algebraic geometry.

\section{PERIODIC $\alpha$-Fractions}

Consider the $N$-periodic $\alpha$-fraction of period $N=2 g+1$

$$
\phi=b_{0}+\frac{\lambda-\alpha_{1}}{b_{1}+\frac{\lambda-\alpha_{2}}{b_{2}+b_{N-1}+\frac{\lambda-\alpha_{N}}{b_{N}+\frac{\lambda-\alpha_{1}}{b_{1}+\frac{\lambda-\alpha_{2}}{b_{2}+}}}}}=\left[b_{0} ; \overline{b_{1}, \ldots, b_{N-1}, b_{N}}\right]_{\alpha}
$$


We see that (at least formally) $\phi$ is the fixed point of the fractional linear transformation

$$
s(\phi)=b_{0}+\frac{a_{1}}{b_{1}+\frac{a_{2}}{b_{2}+\ddots_{b_{N-1}+\frac{a_{N}}{b_{N}^{+\phi}}}}}
$$

with $b_{N}^{*}=b_{N}-b_{0}$ and $a_{k}=\lambda-\alpha_{k}, k=1, . ., N$. The function $s(\phi)$ can be written as $s(\phi)=\frac{P_{N-1} \phi+P_{N}}{Q_{N-1} \phi+Q_{N}}$, where the quantities $P_{k}, Q_{k}$ are determined by the standard recurrence relations (see e.g. [9], page 14):

$$
\begin{gathered}
P_{-1}=1, \quad Q_{-1}=0 \\
P_{0}=b_{0}, \quad Q_{0}=1, \\
P_{k+1}=b_{k+1} P_{k}+a_{k+1} P_{k-1}, \quad Q_{k+1}=b_{k+1} Q_{k}+a_{k+1} Q_{k-1}
\end{gathered}
$$

for $k<N-1$ and

$$
P_{N}=b_{N}^{*} P_{N-1}+a_{N} P_{N-2}, \quad Q_{N}=b_{N}^{*} Q_{N-1}+a_{N} Q_{N-2} .
$$

Thus we have

$$
\phi=\frac{P_{N-1} \phi+P_{N}}{Q_{N-1} \phi+Q_{N}}
$$

which can be written as a quadratic equation

$$
Q_{N-1} \phi^{2}+\left(Q_{N}-P_{N-1}\right) \phi-P_{N}=0 .
$$

It is easy to see from the recurrence relations that $P_{k}$ and $Q_{k}$ are polynomials in $\lambda$ of the form

$$
\begin{gathered}
P_{2 k}=\left(b_{0}+b_{2}+\ldots+b_{2 k}\right) \lambda^{k}+\ldots, \quad P_{2 k+1}=\lambda^{k+1}+\ldots, \\
Q_{2 k}=\lambda^{k}+\ldots, \quad Q_{2 k-1}=\left(b_{1}+b_{3}+\ldots+b_{2 k-1}\right) \lambda^{k-1}+\ldots,
\end{gathered}
$$

for $k \leq g$ and $Q_{2 g+1}=\left(b_{1}+b_{3}+\ldots+b_{2 g-1}+b_{2 g+1}-b_{0}\right) \lambda^{g}+\ldots$, where the dots denote the lower degree terms. Since from (16)

$$
A(\lambda)=Q_{N-1}(\lambda), \quad B(\lambda)=\frac{1}{2}\left(Q_{N}(\lambda)-P_{N-1}(\lambda)\right), \quad C(\lambda)=-P_{N}(\lambda),
$$

the polynomial $A$ is monic of degree $g, C$ is anti-monic of degree $g+1$ and $B$ has degree $g$ or less with the highest term $\beta \lambda^{g}$, where

$$
\beta=-b_{0}+\frac{1}{2} \sum_{k=1}^{N}(-1)^{k+1} b_{k} .
$$

Let us show now that the discriminant $R=B^{2}-A C$ is $\alpha$-admissible. We have

$$
R=\frac{1}{4}\left(Q_{N}-P_{N-1}\right)^{2}+P_{N} Q_{N-1}=\frac{1}{4}\left(P_{N-1}+Q_{N}\right)^{2}+P_{N} Q_{N-1}-P_{N-1} Q_{N} .
$$

We claim that

$$
P_{N} Q_{N-1}-P_{N-1} Q_{N}=\prod_{i=1}^{N}\left(\lambda-\alpha_{i}\right) .
$$

Indeed, the determinant

$$
\left|\begin{array}{cc}
P_{N} & P_{N-1} \\
Q_{N} & Q_{N-1}
\end{array}\right|=\left|\begin{array}{cc}
b_{N} P_{N-1}+a_{N} P_{N-2} & P_{N-1} \\
b_{N} Q_{N-1}+a_{N} Q_{N-2} & Q_{N-1}
\end{array}\right|=
$$




$$
-a_{N}\left|\begin{array}{ll}
P_{N-1} & P_{N-2} \\
Q_{N-1} & Q_{N-2}
\end{array}\right|=\cdots=(-1)^{N} a_{N} a_{N-1} \ldots a_{1}\left|\begin{array}{cc}
b_{0} & 1 \\
1 & 0
\end{array}\right| .
$$

Since $N$ is odd,

$$
\left|\begin{array}{cc}
P_{N} & P_{N-1} \\
Q_{N} & Q_{N-1}
\end{array}\right|=a_{N} a_{N-1} \ldots a_{1}=\prod_{i=1}^{N}\left(\lambda-\alpha_{i}\right)=\mathfrak{A} .
$$

Now by taking $S(\lambda)=\frac{P_{N-1}+Q_{N}}{2}$, which is a polynomial of degree $g$ or less, we see that $R(\lambda)=S^{2}+\mathfrak{A}$, so $R$ is $\alpha$-admissible. This proves the first part of Theorem 1 in the periodic case.

To prove the second part let us introduce the following matrix

$$
M(\lambda)=\left[\begin{array}{cc}
1 & b_{0} \\
0 & 1
\end{array}\right]\left[\begin{array}{cc}
0 & \lambda-\alpha_{1} \\
1 & b_{1}
\end{array}\right] \ldots\left[\begin{array}{cc}
0 & \lambda-\alpha_{N} \\
1 & b_{N}^{*}
\end{array}\right],
$$

with $b_{N}^{*}=b_{N}-b_{0}$. One can check that it can be rewritten also as

$$
M=\left[\begin{array}{cc}
b_{0} & \lambda-\alpha_{1} \\
1 & 0
\end{array}\right] \ldots\left[\begin{array}{cc}
b_{N-1} & \lambda-\alpha_{N} \\
1 & 0
\end{array}\right]\left[\begin{array}{cc}
1 & b_{N}^{*} \\
0 & 1
\end{array}\right] .
$$

The following Lemma explains its importance for our problem.

Lemma 1. Vector $\left(\begin{array}{c}\phi \\ 1\end{array}\right)$ with $\phi=\left[b_{0} ; \overline{b_{1}, \ldots, b_{N-1}, b_{N}}\right]_{\alpha}$ is an eigenvector of the matrix $M(\lambda)$.

The proof follows from the fact that $\phi$ is the fixed point of the fractional linear transformation (13). The product of matrices (18) corresponds to the representation of $s(\phi)$ as a superposition $s_{0} \circ s_{1} \circ \cdots \circ s_{N}(\phi)$, where $s_{0}(\phi)=b_{0}+\phi, s_{k}(\phi)=\frac{\lambda-\alpha_{k}}{b_{k}+\phi}$ for $k=1,2, \ldots, N-1$ and $s_{N}(\phi)=\frac{\lambda-\alpha_{N}}{b_{N}^{*}+\phi}$.

Let $T(\lambda)=\frac{1}{2} \operatorname{tr} M$ be half of the trace of the matrix $M(\lambda)$, which is a polynomial of degree $g$ or less. Note that the determinant of $M$ is equal to $-\mathfrak{A}=-\prod_{i=1}^{N}\left(\lambda-\alpha_{i}\right)$ as it follows immediately from (18).

Lemma 2. The matrix (18) has the form

$$
M(\lambda)=\left[\begin{array}{cc}
T(\lambda)-B(\lambda) & -C(\lambda) \\
A(\lambda) & T(\lambda)+B(\lambda)
\end{array}\right]
$$

where $(A, B, C)$ is the $\alpha$-triple of polynomials corresponding to $\phi$. The discriminant $R=B^{2}-A C$ equals to $T^{2}+\mathfrak{A}$.

Indeed

$$
M(\lambda)=\left[\begin{array}{ll}
P_{N-1}(\lambda) & P_{N}(\lambda) \\
Q_{N-1}(\lambda) & Q_{N}(\lambda)
\end{array}\right]
$$

where $P_{k}, Q_{k}$ are defined above by (14). Now the first claim follows from the relations (17). Taking the determinant of both sides of (20) we have $-\mathfrak{A}=T^{2}-$ $B^{2}+A C$, which implies $B^{2}-A C=T^{2}+\mathfrak{A}$.

Now we need the following result about the factorisation of such matrices. This kind of problems often appears in the theory of discrete integrable systems (see [10] and $[11])$. 
Proposition 1. Let $M(\lambda)$ be a polynomial matrix of the form (20), where $A$ is a monic polynomial of degree $g, C$ is an anti-monic polynomial of degree $g+1, T$ and $B$ are polynomials of degree $g$ or less. Assume also that $\operatorname{det} M(\lambda)=-\prod_{i=1}^{N}\left(\lambda-\alpha_{i}\right)$. Then for an open dense set of such $M$ there exists a unique factorisation of the form

$$
M(\lambda)=\left[\begin{array}{cc}
b_{0} & \lambda-\alpha_{1} \\
1 & 0
\end{array}\right] \ldots\left[\begin{array}{cc}
b_{N-1} & \lambda-\alpha_{N} \\
1 & 0
\end{array}\right]\left[\begin{array}{cc}
1 & b_{N}-b_{0} \\
0 & 1
\end{array}\right] .
$$

The proof is actually effective. We describe the procedure which allows to find $b_{i}$ uniquely assuming at the beginning that the factorisation exists.

Consider the transpose $M^{T}$ of the matrix $M$. For $\lambda=\alpha_{1}$ the matrix $M^{T}(\lambda)$ is degenerate $\left(\right.$ since $\left.\operatorname{det} M^{T}(\lambda)=\operatorname{det} M(\lambda)=-\prod_{i=1}^{N}\left(\lambda-\alpha_{i}\right)\right)$. Find the null-vector $e_{1}=\left(\begin{array}{l}x_{1} \\ y_{1}\end{array}\right)$ of $M^{T}\left(\alpha_{1}\right)$, which is by definition any non-zero vector such that

$$
M^{T}\left(\alpha_{1}\right) e_{1}=0,
$$

or explicitly

$$
\left[\begin{array}{cc}
T\left(\alpha_{1}\right)-B\left(\alpha_{1}\right) & C\left(\alpha_{1}\right) \\
A\left(\alpha_{1}\right) & T\left(\alpha_{1}\right)+B\left(\alpha_{1}\right)
\end{array}\right]\left(\begin{array}{l}
x_{1} \\
y_{1}
\end{array}\right)=\left(\begin{array}{l}
0 \\
0
\end{array}\right) .
$$

It must satisfy the relation

$$
\left[\begin{array}{cc}
b_{0} & 1 \\
0 & 0
\end{array}\right]\left(\begin{array}{l}
x_{1} \\
y_{1}
\end{array}\right)=\left(\begin{array}{l}
0 \\
0
\end{array}\right)
$$

since all other factors are non-degenerate when $\lambda=\alpha_{1}$. This determines $b_{0}$ uniquely as

$$
b_{0}=\frac{T\left(\alpha_{1}\right)-B\left(\alpha_{1}\right)}{A\left(\alpha_{1}\right)}=\frac{C\left(\alpha_{1}\right)}{T\left(\alpha_{1}\right)+B\left(\alpha_{1}\right)} .
$$

Consider now the matrix $M_{1}=\left[\begin{array}{cc}b_{0} & \lambda-\alpha \\ 1 & 0\end{array}\right]^{-1} M(\lambda)$. It is polynomial in $\lambda$ because of the following elementary

Lemma 3. Let $M$ be the polynomial matrix, $\lambda=\alpha$ be a simple root of its determinant and $e=\left(\begin{array}{c}1 \\ -b\end{array}\right)$ be a null vector of $M^{T}(\alpha)$. Then the matrix $\left[\begin{array}{cc}b & \lambda-\alpha \\ 1 & 0\end{array}\right]^{-1} M(\lambda)$ is polynomial.

Indeed, let $M=\left[\begin{array}{cc}X(\lambda) & Y(\lambda) \\ Z(\lambda) & W(\lambda)\end{array}\right]$ then

$$
\left[\begin{array}{cc}
b & \lambda-\alpha \\
1 & 0
\end{array}\right]^{-1} M(\lambda)=\left[\begin{array}{cc}
Z(\lambda) & W(\lambda) \\
\frac{X(\lambda)-b Z(\lambda)}{\lambda-\alpha} & \frac{Y(\lambda)-b W(\lambda)}{\lambda-\alpha}
\end{array}\right] .
$$

From $M^{T}(\alpha)\left[\begin{array}{c}1 \\ -b\end{array}\right]=0$ it follows that $\lambda=\alpha$ is a root of the polynomials $X(\lambda)-$ $b Z(\lambda)$ and $Y(\lambda)-b W(\lambda)$. Therefore these polynomials are divisible by $\lambda-\alpha$, which proves the claim.

Repeat now the procedure by taking $\lambda=\alpha_{2}$ and so on. After $N$ steps we will come to a polynomial matrix

$$
M_{N}(\lambda)=\left[\begin{array}{cc}
b_{N-1} & \lambda-\alpha_{N} \\
1 & 0
\end{array}\right]^{-1} \times \cdots \times\left[\begin{array}{cc}
b_{0} & \lambda-\alpha_{1} \\
1 & 0
\end{array}\right]^{-1} M(\lambda)
$$


with determinant 1. To complete the proof of Proposition 1 we need to show that $M_{N}$ is of the form $\left[\begin{array}{cc}1 & b_{N}^{*} \\ 0 & 1\end{array}\right]$.

Recall that the matrix $M(\lambda)$ is of the form $\left[\begin{array}{cc}a_{0} \lambda^{g}+\ldots & \lambda^{g+1}+\ldots \\ \lambda^{g}+\ldots & d_{0} \lambda^{g}+\ldots,\end{array}\right]$, where the dots mean terms of lower degree, and the coefficients $a_{0}$ and $d_{0}$ may be zero. It is easy to show that the matrix $M_{2}(\lambda)=\left[\begin{array}{cc}b_{1} & \lambda-\alpha_{2} \\ 1 & 0\end{array}\right]^{-1}\left[\begin{array}{cc}b_{0} & \lambda-\alpha_{1} \\ 1 & 0\end{array}\right]^{-1} M(\lambda)$ is of the form $\left[\begin{array}{cr}a_{2} \lambda^{g-1}+\ldots & \lambda^{g}+\ldots \\ \lambda^{g-1}+\ldots & d_{2} \lambda^{g-1}+\ldots\end{array}\right]$ and by induction $M_{2 k}(\lambda)$ is of the form $\left[\begin{array}{cc}a_{k} \lambda^{g-k}+\ldots & \lambda^{g-k+1}+\ldots \\ \lambda^{g-k}+\ldots & d_{k} \lambda^{g-k}+\ldots\end{array}\right]$. Therefore the matrix $M_{N-1}$ is of the form $\left[\begin{array}{cc}a_{g} & \lambda+c \\ 1 & d_{g}\end{array}\right]$ where $a_{g}, c, d_{g}$ are constant. The matrix $M_{N}=\left[\begin{array}{cc}b_{N-1} & \lambda-\alpha_{N} \\ 1 & 0\end{array}\right]^{-1} M_{N-1}$ equals to $\left[\begin{array}{cc}1 & d_{g} \\ \frac{a_{g}-b_{N-1}}{\lambda-\alpha_{N}} & \frac{\lambda+c-b_{N-1} d_{g}}{\lambda-\alpha_{N}}\end{array}\right]$. Since $M_{N}$ is a polynomial matrix, we have $a_{g}=b_{N-1}$ and $b_{N-1} d_{g}-c=\alpha_{N}$. Thus $M_{N}$ has the required form.

We see that the procedure will not work only if at some stage the first component of the null vector of $M_{k}^{T}\left(\alpha_{k+1}\right)$ vanishes. Clearly this happens only for a closed algebraic subset of codimension 1 , so for generic triples $(A, B, C)$ the matrix decomposition exists and is unique. This completes the proof of Proposition 1.

Now we are ready to finish the proof of Theorem 1 in the periodic case. Let $(A, B, C)$ be an $\alpha$-triple, then by definition there exists a polynomial matrix $S$ of degree $g$ or less such that the discriminant $R=B^{2}-A C$ is equal to $S^{2}+\mathfrak{A}$. Clearly the polynomial $S$ is defined up to a sign. Consider two corresponding matrices $M$ given by (20) with $T(\lambda)= \pm S(\lambda)$. Each of them generically has a unique factorisation given by Proposition 1 . One can easily check that this gives two $N$-periodic $\alpha$-fraction representations of the corresponding function $\phi(\lambda)=$ $\frac{-B(\lambda)+\sqrt{R(\lambda)}}{A(\lambda)}$ and thus completes the proof in this case.

\section{PURE PERIODIC $\alpha$-FraCtions}

Let now $\phi=\left[\overline{b_{0}, b_{1}, \ldots, b_{N-1}}\right]_{\alpha}$ be a pure periodic $\alpha$-fraction. This is a particular case of the previous situation with $b_{0}=b_{N}$. But since the corresponding $b_{N}^{*}=$ $b_{0}-b_{N}=0$, this case is actually degenerate and needs a special consideration.

First of all as before $\phi$ satisfies the relation

$$
\phi=\frac{P_{N-1} \phi+P_{N}}{Q_{N-1} \phi+Q_{N}}
$$

where $P_{k}, Q_{k}$ satisfy the relations (14), but now because $b_{N}^{*}=0$ we have

$$
P_{N}=\left(\lambda-\alpha_{N}\right) P_{N-2}, \quad Q_{N}=\left(\lambda-\alpha_{N}\right) Q_{N-2} .
$$

Now from (17) we have

$A(\lambda)=Q_{N-1}(\lambda), B(\lambda)=\frac{1}{2}\left(\left(\lambda-\alpha_{N}\right) Q_{N-2}(\lambda)-P_{N-1}(\lambda)\right), C(\lambda)=-\left(\lambda-\alpha_{N}\right) P_{N-2}$.

Since $Q_{N-1}$ and $P_{N-2}$ are monic this shows that $A, B, C$ satisfy the property (1) of Theorem 1 with the additional condition $C\left(\alpha_{N}\right)=0$. The proof of the second property ( $\alpha$-admissibility of $R$ ) goes unchanged. 
Now as in the previous case in order to find the pure periodic $\alpha$-fraction one should factorise the matrix

$$
M(\lambda)=\left[\begin{array}{cc}
T(\lambda)-B(\lambda) & -C(\lambda) \\
A(\lambda) & T(\lambda)+B(\lambda)
\end{array}\right]
$$

as the product

$$
\left[\begin{array}{cc}
b_{0} & \lambda-\alpha_{1} \\
1 & 0
\end{array}\right] \cdots\left[\begin{array}{cc}
b_{N-1} & \lambda-\alpha_{N} \\
1 & 0
\end{array}\right] \text {. }
$$

The main difference is that in the pure periodic case the trace $T(\lambda)$ of the matrix $M(\lambda)$, which was known before only up to a sign, now is determined uniquely by the condition

$$
T\left(\alpha_{N}\right)=-B\left(\alpha_{N}\right) .
$$

Indeed $T\left(\alpha_{N}\right)=\frac{1}{2}\left(P_{N-1}\left(\alpha_{N}\right)+Q_{N}\left(\alpha_{N}\right)\right)=\frac{1}{2}\left(P_{N-1}\left(\alpha_{N}\right)\right)=-B\left(\alpha_{N}\right)$ since $Q_{N}\left(\alpha_{N}\right)=0$. If $B\left(\alpha_{N}\right) \neq 0$, which is a generic case, this determines $T(\lambda)$ uniquely (and thus the matrix $M)$ by a triple $(A, B, C)$. This completes the proof of Theorem 1.

Example. Consider the simplest case $N=1, g=0$. Then the $\alpha$-triples have the form

$$
A=1, \quad B=\beta, \quad C=-(\lambda+\gamma)
$$

with arbitrary $\beta, \gamma \in \mathbb{C}$, so that in the periodic case the corresponding $\phi$ have a general form

$$
\phi=-\beta+\sqrt{\lambda+\gamma}
$$

In this particular case this can be easily seen directly. Indeed $\phi=\left[b_{0}, \overline{b_{1}}\right]_{\alpha}$ satisfies the quadratic equation

$$
\phi^{2}+\left(b_{1}^{*}-b_{0}\right) \phi-\left(\lambda-\alpha_{1}+b_{0} b_{1}^{*}\right)=0,
$$

where $b_{1}^{*}=b_{1}-b_{0}$. Thus to find a periodic continued $\alpha$-fraction expansion of $(24)$ one should solve the system of equations

$$
b_{1}^{*}-b_{0}=2 \beta, \quad b_{0} b_{1}^{*}=\alpha_{1}+\gamma,
$$

which has two solutions

$$
b_{0}=-\beta \pm \sqrt{\beta^{2}+\alpha_{1}+\gamma}, \quad b_{1}^{*}=\beta \pm \sqrt{\beta^{2}+\alpha_{1}+\gamma} .
$$

One can easily check that these two solutions correspond to two solutions of the factorisation problem from the previous section.

In the pure periodic case by the additional condition (8) we have $C=-\left(\lambda-\alpha_{1}\right)$, so $\gamma=-\alpha_{1}$ and the general form of $\phi$ is

$$
\phi=-\beta+\sqrt{\lambda-\alpha_{1}} .
$$

In that case we have also $b_{1}^{*}=b_{1}-b_{0}=0$, so the system (25) reduces to just one equation $b_{0}=-2 \beta$, which determines the pure periodic $\alpha$-fraction expansion of (26) uniquely in agreement with our previous consideration. 


\section{ACTION OF $\mathbb{Z}_{2} \times S_{N}$}

A surprising corollary of Theorem 1 is the invariance of the set of $N$-periodic $\alpha$-fractions under the permutations $\sigma \in S_{N}$ of the set $\alpha$ :

$$
\sigma(\alpha)_{k}=\alpha_{\sigma(k)} .
$$

This is not obvious from the very beginning and in fact is not true in the pure periodic case.

In this section we explain how to use this symmetry to describe all $2 N$ ! periodic $\alpha$-fractions for a given algebraic function $\phi$. In fact, the full symmetry group is the product $G=\mathbb{Z}_{2} \oplus S_{N}$, where $\mathbb{Z}_{2}$ is generated by the involution $\varepsilon$ interchanging two different $\alpha$-fraction expansions with the same order of the parameters $\alpha_{i}$.

The action of this group is described by Theorem 2 . We are going to prove it now.

Recall that $\pi \in S_{N}$ is the permutation, which reverses the order $1,2, \ldots, N-1, N$ to $N, N-1, \ldots, 2,1$, and the involution $\sigma_{k}$ swaps $k$ and $k+1$ leaving the rest fixed.

Let us start with the action of $\sigma_{k}$ first. Let us introduce (assuming that $b_{k} \neq 0$ )

$$
\tilde{b}_{k-1}=b_{k-1}+\frac{\alpha_{k+1}-\alpha_{k}}{b_{k}}, \quad \tilde{b}_{k+1}=b_{k+1}-\frac{\alpha_{k+1}-\alpha_{k}}{b_{k}}, \quad k=1, \ldots, N-1 .
$$

One can check directly the following matrix identity:

$$
\begin{aligned}
& {\left[\begin{array}{cc}
b_{k-1} & \lambda-\alpha_{k} \\
1 & 0
\end{array}\right]\left[\begin{array}{cc}
b_{k} & \lambda-\alpha_{k+1} \\
1 & 0
\end{array}\right]\left[\begin{array}{cc}
b_{k+1} & \lambda-\alpha_{k+2} \\
1 & 0
\end{array}\right]=} \\
& {\left[\begin{array}{cc}
\tilde{b}_{k-1} & \lambda-\alpha_{k+1} \\
1 & 0
\end{array}\right]\left[\begin{array}{cc}
b_{k} & \lambda-\alpha_{k} \\
1 & 0
\end{array}\right]\left[\begin{array}{cc}
\tilde{b}_{k+1} & \lambda-\alpha_{k+2} \\
1 & 0
\end{array}\right] .}
\end{aligned}
$$

Similarly for $k=N-1$ we have $\left[\begin{array}{cc}b_{N-2} & \lambda-\alpha_{N-1} \\ 1 & 0\end{array}\right]\left[\begin{array}{cc}b_{N-1} & \lambda-\alpha_{N} \\ 1 & 0\end{array}\right]\left[\begin{array}{cc}1 & b_{N}-b_{0} \\ 0 & 1\end{array}\right]=$ $\left[\begin{array}{cc}\tilde{b}_{N-2} & \lambda-\alpha_{N} \\ 1 & 0\end{array}\right]\left[\begin{array}{cc}b_{N-1} & \lambda-\alpha_{N-1} \\ 1 & 0\end{array}\right]\left[\begin{array}{cc}1 & \tilde{b}_{N}-b_{0} \\ 0 & 1\end{array}\right]$. Taking into account the results of the previous section we see that the action of $\sigma_{k}$ is indeed given by the formula (9).

To prove the remaining part of Theorem 2 recall that $\phi=\left[b_{0} ; \overline{b_{1}, \ldots, b_{N-1}, b_{N}}\right]_{\alpha}$ is the fixed point of the fractional linear transformation (13), and therefore it is the fixed point of its inverse, which as one can easily check is given by

$$
s^{-1}(\phi)=-b_{N}+b_{0}+\frac{a_{N}}{-b_{N-1}+\frac{a_{N-1}}{-b_{N-2}+\ddots_{-b_{1}+\frac{a_{1}}{-b_{0}+\phi}}}} .
$$

Thus

$$
\phi=\left[b_{0}-b_{N} ; \overline{-b_{N-1}, \ldots,-b_{1},-b_{N}}\right]_{\alpha_{N}, \ldots, \alpha_{1}, \alpha_{0}}
$$

is a periodic $\alpha$-fraction corresponding to the sequence $\pi(\alpha)=\alpha_{N}, \ldots, \alpha_{1}, \alpha_{0}$. Now note that the trace of the corresponding matrix (19) is equal to $P_{N-1}+Q_{N}=$ $\left(b_{1}+b_{2}+\cdots+b_{N}\right) \lambda^{g}+\ldots$ (in the notations of the previous section). If we replace $b_{1}, \ldots, b_{n}$ by $-b_{N-1}, \ldots,-b_{1},-b_{N}$ its highest coefficient clearly changes sign. This means that the new periodic $\alpha$-fraction (28) corresponds to the action of the element $\varepsilon \pi \in G$. Since $\varepsilon \pi$ and $\sigma_{k}$ generate the group $G$ we have described the full action.

In the pure periodic case because of the additional condition $C\left(\alpha_{N}\right)=0$ the symmetry group is reduced to $S_{N-1}$, permuting $\alpha_{i}$ with $i=1, \ldots, N-1$. This 
group is generated by $\sigma_{k}$ with $k=1, \ldots, N-2$ with the action given by the same formula (9). Theorem 2 is proved.

Example. Let $N=3, g=1, \alpha=(1,3,4)$ and

$$
\phi=\frac{3 x-7+\sqrt{4 x^{3}-31 x^{2}+62 x+1}}{2(x-6)} .
$$

We have $A(x)=x-6, \quad B(x)=-\frac{1}{2}(3 x-7), \quad C(x)=-x^{2}+4 x-2$ and $R(x)=$ $\frac{1}{4}\left(4 x^{3}-31 x^{2}+62 x+1\right)=\left(\frac{x-7}{2}\right)^{2}+(x-1)(x-3)(x-4)$ is $\alpha$-admissible.

To each permutation of the sequence $(1,3,4)$ we have the following two periodic continued $\alpha$-fraction representations of $\phi$ :

$$
\begin{aligned}
& \phi=[1 ; \overline{-3,1,3}]_{1,3,4}=\left[-\frac{1}{5} ; \overline{-\frac{15}{6}, \frac{6}{5}, \frac{3}{10}}\right]_{1,3,4} \\
& \phi=[1 ; \overline{-2,1,2}]_{1,4,3}=\left[-\frac{1}{5} ; \overline{-\frac{5}{3}, \frac{6}{5},-\frac{8}{15}}\right]_{1,4,3} \\
& \phi=\left[\frac{1}{3} ; \overline{-3, \frac{5}{3}, \frac{7}{3}}\right]_{3,1,4}=\left[-1 ; \overline{-\frac{15}{6}, 2,-\frac{1}{2}}\right]_{3,1,4} \\
& \phi=\left[\frac{1}{3} ; \overline{-\frac{6}{5}}, \frac{5}{3}, \frac{8}{15}\right]_{3,4,1}=[-1 ; \overline{-1,2,-2}]_{3,4,1} \\
& \phi=\left[-\frac{1}{2} ; \overline{-\frac{6}{5}}, \frac{15}{6},-\frac{3}{10}\right]_{4,3,1}=[-2 ; \overline{-1,3,-3}]_{4,3,1} \\
& \phi=\left[-\frac{1}{2} ; \overline{-2, \frac{15}{6}, \frac{1}{2}}\right]_{4,1,3}=\left[-2 ; \overline{-\frac{5}{3}, 3,-\frac{7}{3}}\right]_{4,1,3} .
\end{aligned}
$$

Once one of them is known we can find the rest using the action of the group $G$ described above.

\section{Relation With the AFFine hyperelliptic Jacobi VARIETIES}

In this section we will discuss the geometric aspects of the periodic $\alpha$-fractions. Let us first note that strictly speaking a function

$$
\phi(\lambda)=\frac{-B(\lambda)+\sqrt{R(\lambda)}}{A(\lambda)}
$$

is not a function of $\lambda \in \mathbb{C}$, but a function on the corresponding hyperelliptic curve $\Gamma(R)$ given by the equation

$$
\mu^{2}=R(\lambda)
$$

This curve has a natural involution $\tau:(\lambda, \mu) \rightarrow(\lambda,-\mu)$, interchanging the branches of the square root.

Let us fix now an $\alpha$-admissible polynomial $R(\lambda)$ and assume that all its roots are distinct, so that the corresponding hyperelliptic curve $\Gamma_{R}$ is non-singular and has genus $g$. Let us ask the following natural question: which functions on $\Gamma_{R}$ admit periodic $\alpha$-fractions ? According to Theorem 1 the variety of such functions is birationally equivalent to the variety $M_{R}^{\alpha}$ of the triples of polynomials $(A, B, C)$ such that

$$
A(\lambda)=\lambda^{g}+\ldots, C(\lambda)=-\lambda^{g+1}+\ldots, \operatorname{deg} B \leq g,
$$


satisfying the relation

$$
B^{2}(\lambda)-A(\lambda) C(\lambda)=R(\lambda)
$$

with given $R$. The triples satisfying conditions (30) form an affine space $\mathbb{C}^{3 g+2}$, while the relation (31) is equivalent to $2 g+1$ algebraic equations on the coefficients of $A, B, C$. Thus $M_{R}^{\alpha}$ is an affine algebraic variety.

We claim that $M_{R}^{\alpha}$ is nothing else but the product $J\left(\Gamma_{R}\right)^{a f f} \times \mathbb{C}$, where $J\left(\Gamma_{R}\right)^{\text {aff }}$ is the affine part of the Jacobi variety of the corresponding hyperelliptic curve (29). The proof is essentially due to Jacobi [6], who found the following elementary description of the hyperelliptic Jacobi variety. We follow here Mumford's lectures $[7]$.

The affine Jacobi variety $J\left(\Gamma_{R}\right)^{a f f}$ is defined as the set of the positive divisors $D=P_{1}+\cdots+P_{g}$, where $P_{1}, \ldots, P_{g}$ are points on the affine curve (29) (not necessarily distinct) such that $P_{i} \neq \tau\left(P_{j}\right)$ for any $i \neq j$.

Consider the following Jacobi triples $(U, V, W)$ of polynomials in $\lambda$, where $U$ and $W$ are monic polynomials of degree $g$ and $g+1$ respectively, $V$ is a polynomial of degree less or equal than $g-1$, such that the following relation is satisfied

$$
V^{2}(\lambda)+U(\lambda) W(\lambda)=R(\lambda)
$$

We denote the corresponding variety $N_{R}$.

Theorem (Jacobi). There is a natural bijection between the set $N_{R}$ of the Jacobi triples and the affine Jacobi variety $J\left(\Gamma_{R}\right)^{\text {aff }}$.

We sketch here the proof, which is actually not difficult. Let $D=P_{1}+\cdots+P_{g}$ be a divisor from $J\left(\Gamma_{R}\right)^{a f f}$. Let us assume at the beginning that all points $P_{i} \in \Gamma_{R}$ are distinct. We would like to associate with $D$ a Jacobi triple $(U, V, W)$. This can be done as follows.

Let $\left(\mu_{i}, \lambda_{i}\right)$ be coordinates of the points $P_{i}$. Then the polynomial $U$ is defined as

$$
U(\lambda)=\prod_{i=1}^{g}\left(\lambda-\lambda_{i}\right)
$$

The polynomial $V$ is defined by the Lagrange interpolation formula from the conditions $V\left(\lambda_{i}\right)=\mu_{i}, i=1, \ldots, g$ as

$$
V(\lambda)=\sum_{i=1}^{g} \mu_{i} \frac{\prod_{j \neq i}\left(\lambda-\lambda_{j}\right)}{\prod_{j \neq i}\left(\lambda_{i}-\lambda_{j}\right)}
$$

(note that by assumptions all $\lambda_{i}$ are distinct). Now $W$ is defined uniquely by the relation (32). It is a polynomial because by construction $R-V^{2}$ vanishes at all the zeroes of $U(\lambda)$. If some of the points $P_{i}$ collide one should use a natural generalisation involving also the derivatives (see [7] for details). This defines a natural map from $J\left(\Gamma_{R}\right)^{\text {aff }}$ to $N_{R}$.

The inverse map is also quite natural: the $\lambda$-coordinates of $P_{i}$ are given by the zeroes of $U(\lambda)$ while the $\mu$-coordinates are the values of the polynomial $V$ at these zeroes. The fact that the corresponding points $P_{i}$ belong to $\Gamma_{R}$ follows from the relation (32).

Now let us return to our set $M_{R}^{\alpha}$. To every Jacobi triple $(U, V, W)$ and complex number $\beta \in \mathbb{C}$ we can relate a unique $\alpha$-triple $(A, B, C)$ defined by

$$
A=U, \quad B=V+\beta U, \quad C=-W+2 \beta V+\beta^{2} U .
$$


Indeed

$$
B^{2}-A C=(V+\beta U)^{2}-U\left(-W+2 \beta V+\beta^{2} U\right)=V^{2}+U W=R .
$$

Conversely, for a given $\alpha$-triple $(A, B, C)$ there exists a unique pair $((U, V, W), \beta)$ where $\beta$ is the coefficient of $\lambda^{g}$ in $B$ and

$$
U=A, V=B-\beta A, W=-C+2 \beta B-\beta^{2} A
$$

is a Jacobi triple.

In the pure periodic case we have a well-defined 2:1 map $f: P_{R}^{\alpha} \rightarrow J\left(\Gamma_{R}\right)^{\text {aff }}$ defined by the same formulae (33). Indeed for a given Jacobi triple $(U, V, W)$ there are generically $2 \alpha$-triples $(A, B, C)$ given by (34), where $\beta$ must satisfy the quadratic equation

$$
U\left(\alpha_{N}\right) \beta^{2}+2 V\left(\alpha_{N}\right) \beta-W\left(\alpha_{N}\right)=C\left(\alpha_{N}\right)=0,
$$

so that $\beta=-\frac{V\left(\alpha_{N}\right) \pm \sqrt{R\left(\alpha_{N}\right)}}{U\left(\alpha_{N}\right)}$ if $U\left(\alpha_{N}\right) \neq 0$ or $\beta=\frac{W\left(\alpha_{N}\right)}{2 V\left(\alpha_{N}\right)}$ if $U\left(\alpha_{N}\right)=0$ and $V\left(\alpha_{N}\right) \neq 0$. Note that if both $U\left(\alpha_{N}\right)=0$ and $V\left(\alpha_{N}\right)=0$, then $R\left(\alpha_{N}\right)=0$ which we have excluded.

In other words, to any divisor of degree $g$ of the form $D=\sum_{i=1}^{g} P_{i}$, we construct the polynomial $A(\lambda)$ in the same way as $U(x)$. The condition $C\left(\alpha_{N}\right)=0$ implies $B\left(\alpha_{N}\right)=\sqrt{R\left(\alpha_{N}\right)}$, which means that the polynomial $B(\lambda)$ is now required to pass through the $g$ points $P_{i}$ plus the additional point $P\left(\alpha_{N}, \sqrt{R\left(\alpha_{N}\right)}\right) \in \Gamma_{R}$. Generically there are two such points depending on the choice of the square root, which determines the corresponding $B(\lambda)$ and thus the $\alpha$-triple uniquely.

Conversely, to any $\alpha$-triple $(A, B, C)$ with $C\left(\alpha_{N}\right)=0$ we can associate a divisor $D$ and a point $P \in \Gamma_{R}$ such that $\Lambda(P)=\alpha_{N}$, where $\Lambda: \Gamma_{R} \rightarrow \mathbb{C}$ be the projection defined by $\Lambda(\lambda, \mu)=\lambda$.

Let us discuss now the corresponding meromorphic functions $\phi$ on $\Gamma_{R}$.

Let $D=P_{1}+\ldots P_{g}, P_{i} \in \Gamma_{R}^{a f f}$ be a positive non-special divisor, $L\left(D+P_{\infty}\right)$ be the corresponding linear space of meromorphic functions with poles at most at $P_{1}, \ldots, P_{g}$ and $P_{\infty}$. It has dimension 2. Indeed by the classical Riemann-Roch theorem [5]

$$
\operatorname{dim} L\left(D+P_{\infty}\right)=2+\operatorname{dim} \Omega\left(D+P_{\infty}\right),
$$

where $\Omega\left(D+P_{\infty}\right)$ is the linear space of holomorphic 1-forms $\omega$ on $\Gamma_{R}$ with zeroes at $P_{1}, \ldots, P_{g}$ and $P_{\infty}$. Any holomorphic 1-form on the hyperelliptic curve $\Gamma_{R}$ has a form

$$
\omega=\frac{P(\lambda) d \lambda}{\sqrt{R(\lambda)}}
$$

where $P(\lambda)$ is a polynomial of degree less than or equal to $g-1$. Since $D$ is nonspecial, such $\omega$ must be identically zero so that $\operatorname{dim} \Omega\left(D+P_{\infty}\right)=0$ and $\operatorname{dim} L(D+$ $\left.P_{\infty}\right)=2$.

Consider the functions from the linear space $L\left(D+P_{\infty}\right)$ which are equivalent to $\sqrt{\lambda}$ at infinity; these functions differ from each other by additive constant. One can easily see that up to this freedom they have the form (4) given by the Jacobi construction above. Note the formulae (33) corresponds to the shift $\phi \rightarrow \phi+\beta$. In the pure periodic case $\phi$ has a zero at one of two points of $\Gamma_{R}$ with $\lambda=\alpha_{N}$, which reduces the shift to two values. This completes the proof of Theorem 3 . 


\section{ACKNOWLEDGEMENTS}

We are very grateful to Vassilis Papageorgiou for the numerous stimulating discussions.

This work has been partially supported by the EPSRC and by the European Union through the FP6 Marie Curie RTN ENIGMA (Contract number MRTNCT-2004-5652) and the ESF programme MISGAM.

\section{REFERENCES}

[1] A.P. Veselov, A.B. Shabat Dressing chain and spectral theory of Schrödinger operators. Funct. Anal. Appl., 22 (1993).

[2] M.-P. Grosset, V. Papageorgiou, A.P. Veselov Discrete KdV equation and Yang-Baxter correspondences. In preparation

[3] A.J. van der Poorten and X.C. Tran Quasi-elliptic integrals and periodic continued fractions. Monatsch. Math. 131(2) (2000), 155-169.

[4] W. J. LeVeque Fundamentals of Number Theory. Dover Publ., New York, 1977.

[5] Ph. Griffiths, J. Harris Principles of Algebraic Geometry. John Wiley and Sons, New York, 1978.

[6] C.G.J. Jacobi Zur Theorie der Variation-Rechnung und der differential Gleihungen. J. Reine Angew. Math. 32 (1846).

[7] D. Mumford Tata lectures on Theta: Volume II: Jacobian theta functions and differential equations, Birkhäuser Boston, 1984.

[8] B.A. Dubrovin, S.P. Novikov A periodic problem for the Korteweg-de Vries and SturmLiouville equations. Their connection with algebraic geometry. Dokl. Akad. Nauk SSSR, 219, N 3 (1974), 531- 534.

[9] H.S. Wall Analytic theory of continued fractions, D. Van Nostrand Company, inc., 1948.

[10] J. Moser, A.P. Veselov Discrete versions of some classical integrable systems and factorisation of matrix polynomials. Comm. Math. Phys. 139 (1991), 217-243.

[11] V.E. Adler, A.P. Veselov Cauchy problem for integrable discrete equations on quad-graphs. Acta Appl. Math. 84 (2004), 237-262.

Department of Mathematical Sciences, Loughborough University, Loughborough, LEICESTERSHIRE, LE11 3TU, UK

E-mail address: M.Grosset@lboro.ac.uk

Department of Mathematical Sciences, Loughborough University, Loughborough, Leicestershire, Le11 3TU, UK and Landau Institute for Theoretical Physics, Moscow, RUsSiA

E-mail address: A.P.Veselov@lboro.ac.uk 